\title{
Das Bundesverfassungsgericht als europäischer Gesetzgeber oder als Motor der Union?
}

\author{
Von Thomas M.J. Möllers und Katharina Redcay, Augsburg*
}

Mit der Eilentscheidung zum ESM-Vertrag zeigte das Bundesverfassungsgericht erneut, dass es eine aktive Rolle in Europa spielen will und sich nicht ohne weiteres in die dritte Reihe zurückdrängen lässt, wenn das Recht der EU und sein Stellenwert im deutschen Rechtssystem betroffen sind. Bereits in älteren Entscheidungen formulierte es den seiner Ansicht nach einzig richtigen zukünftigen Kurs der Europäischen Union und konnte meist beobachten, dass dieser dann auch eingeschlagen wurde. Das Bundesverfassungsgericht prägt dadurch das europäische Recht, wobei es nicht immer die Grenzen zulässiger Rechtsfortbildung achtet und in die Aufgabenbereiche von europäischem Gesetzgeber und Europäischem Gerichtshof eingreift.

\section{Einleitung}

Die jüngste Entscheidung des Bundesverfassungsgerichts über den Eilantrag zum Europäischen Stabilitätsmechanismus (ESM) war von den Märkten lange erwartet worden und wurde dann ganz überwiegend mit Kurssteigerungen an den Börsen gefeiert. ${ }^{1}$ Im Ergebnis hält das Gericht den ESM-Vertrag zwar mit der deutschen Verfassung für vereinbar. Mit deutlichen Worten schreibt es der Bundesregierung aber vor, mittels eines Vorbehalts völkerrechtlich sicherzustellen, dass die deutsche Zahlungsverpflichtung durch den ESM nicht über 190 Mrd. EUR ausgedehnt werden darf bzw. in diesem Fall keine deutsche Bindung besteht. ${ }^{2}$ Dieser Vorbehalt wurde inzwischen auch erklärt. ${ }^{3}$

Des Weiteren nimmt es auch zu den Aktivitäten der EZB Stellung und erklärt:

, [...] ein Erwerb von Staatsanleihen am Sekundärmarkt durch die Europäische Zentralbank, der auf von den Kapitalmärkten unabhängige Finanzierung der Haushalte der Mitgliedstaaten zielte, ist als Umgehung des Verbotes monetärer Haushaltsfinanzierung ebenfalls untersagt. "4

* Prof. Dr. Thomas M.J. Möllers ist Inhaber des Lehrstuhls für Bürgerliches Recht, Wirtschaftsrecht, Europarecht, Internationales Privatrecht und Rechtsvergleichung der Universität Augsburg, Geschäftsführender Direktor des Center for European Legal Studies (CELOS) sowie Inhaber eines Jean-Monnet-Lehrstuhls. Katharina Redcay ist wissenschaftliche Mitarbeiterin am Lehrstuhl für Bürgerliches Recht, Wirtschaftsrecht, Europarecht, Intemationales Privatrecht und Rechtsvergleichung der Universität Augsburg.

1 S. etwa D. Mohr, Nur kurze Erleichterung nach dem ESM-Entscheid, FAZ v. 13.9.2012, S. 24; D. Neuerer/G. Watzlawek, Freie Fahrt für die Euro-Retter, www.handelsblatt.com v. 12.9.2012; N.N., Wallstreet schaut nach ESM-Urteil auf die FED, www.handelsblatt.com v. 12.9.2012; N.N., Aktienmarkt nach ESM-Urteil deutlich fester, www.faz.net v. 12.9.2012.

2 BVerfG, Urt. v. 12.9.2012, NJW 2012, 3145, 3153, Rn. 253 ff. - ESM.

3 Der ESM-Vertrag trat damit am 8.10.2012 in Kraft, s. H. Kafsack, Eurostaaten setzen ESM in Kraft, FAZ v. 9.10.2012, S. 1 f. Für den Wortlaut der Übereinkunft s. BT-Drucks. 17/10767 v. 25.9.2012, S. 5.

4 BVerfG, Urt. v. 12.9.2012, NJW 2012, 3145, 3156, Rn. 278-ESM. 
Die Methodik des ESM-Urteils entspricht in einem wichtigen Punkt der anderer europarechtlicher Urteile des Bundesverfassungsgerichts: Bei allen diesen Urteilen fällt auf, dass das Gericht die europäische Entscheidung bzw. Änderung zwar in der Regel akzeptiert, dann aber aufzeigt, welche Vorgaben in Zukunft zu erfüllen sind, damit bestimmte Zustände nicht für rechtswidrig erklärt werden müssen. Nicht zuletzt im Hinblick auf die in naher Zukunft zu erwartenden Entscheidungen über den ESM-Vertrag im Hauptsacheverfahren und die EZB lohnt es sich zu diskutieren, ob das Bundesverfassungsgericht, wie der Titel dieses Beitrags andeutet, tatsächlich und in doppelter Hinsicht seine Kompetenzen überschreitet und sich zum europäischen Richter oder gar zum europäischen Gesetzgeber aufschwingt. Dazu sind zunächst einige der wichtigsten Entscheidungen des Bundesverfassungsgerichts zum Europarecht vorzustellen (B.), bevor die Frage der Kompetenzüberschreitung methodisch untersucht wird (C.).

\section{Die wichtigsten europarechtlichen Entscheidungen des BVerfG und ihre Aufnahme im Unionsrecht}

\section{Zum Grundrechtsschutz in der Gemeinschaft}

\section{a) Die Solange-I-Entscheidung}

$\mathrm{Zu}$ den frühen Entscheidungen des Bundesverfassungsgerichts zum Europarecht gehören die beiden so genannten Solange-Entscheidungen. ${ }^{5}$ Sie zeigen, wie das Bundesverfassungsgericht den europäischen Integrationsprozess ankurbelte. In der Solange-I-Entscheidung vom 29. Mai 1974 forderte das Bundesverfassungsgericht ganz dezidiert einen geschriebenen Grundrechtskatalog auf europäischer Ebene. Der entsprechende Leitsatz lautete:

",Solange der Integrationsprozeß der Gemeinschaft nicht so weit fortgeschritten ist, daß das Gemeinschaftsrecht auch einen von einem Parlament beschlossenen und in Geltung stehenden formulierten Katalog von Grundrechten enthält, der dem Grundrechtskatalog des Grundgesetzes adäquat ist, ist nach Einholung der in Art. 177 EWGV geforderten Entscheidung des Europäischen Gerichtshofes die Vorlage eines Gerichts der Bundesrepublik Deutschland an das Bundesverfassungsgericht im Normenkontrollverfahren zulässig und geboten, wenn das Gericht die für es entscheidungserhebliche Vorschrift des Gemeinschaftsrechts in der vom Europäischen Gerichtshof gegebenen Auslegung für unanwendbar hält, weil und soweit sie mit einem der Grundrechte des Grundgesetzes kollidiert. "6

5 BVerfG, Urt. v. 29.5.1974, BVerfGE 37, 271 -Solange I; BVerfG, Urt. v. 22. 10.1986, BVerfGE 73, 339-Solange
II.

6 BVerfG, Urt. v. 29.5.1974, BVerfGE 37, 271, Ls. sowie 285 - Solange I. 


\section{b) Einführung von Grundrechten auf europäischer Ebene}

Nur wenige Tage vor der Solange-I-Entscheidung hatte der EuGH in der NoldEntscheidung ${ }^{7}$ gemeinschaftsrechtliche Grundrechte aus den ,gemeinsamen Verfassungsüberlieferungen der Mitgliedstaaten" ${ }^{8}$ entwickelt. Darüber hinaus berief sich der EuGH auf die EMRK. ${ }^{9}$ Die Ausführungen blieben naturgemäß noch recht vage.

In den folgenden Jahren präzisierte der EuGH den Grundrechtsschutz. ${ }^{10}$ In der Solange-II-Entscheidung vollzog das Bundesverfassungsgericht dann eine 180-GradWende, indem es feststellte, dass der europäische Grundrechtsschutz inzwischen in Konzeption, Inhalt und Wirkungsweise im Wesentlichen den deutschen Grundrechten entspreche. ${ }^{11}$ Es sei folglich für die Überprüfung der Anwendbarkeit von abgeleiteten Gemeinschaftsrechtsakten nicht mehr zuständig. ${ }^{12}$

Das Gericht nahm damit von der Forderung nach einem geschriebenen Grundrechtskatalog scheinbar Abstand und akzeptierte die ungeschriebenen Grundrechte des EuGH. Zuvor hatten das Europäische Parlament, der Rat und die Kommission die Grundrechte ausdrücklich anerkannt. ${ }^{13}$ Die weitergehende Forderung des Bundesverfassungsgerichts nach einem geschriebenen Grundrechtskatalog wurde vom Europäischen Parlament aufgenommen. ${ }^{14}$ Der 1992 mit dem Vertrag von Maastricht einge führte EU-Vertrag ${ }^{15}$ stellte in Art. F klar, die Union achte die Grundrechte der EMRK und jene der gemeinsamen Verfassungsüberlieferungen der Mitgliedstaaten. Der Forderung nach geschriebenen Grundrechten wurde erst zehn Jahre später entsprochen: Die Europäische Charta der Grundrechte wurde im Rahmen der Vorbereitungen einer Europäischen Verfassung 1999 vom Europäischen Rat initiiert und im Jahr 2000 feierlich proklamiert. ${ }^{16}$ Seit Inkrafttreten des Vertrags von Lissabon

7 EuGH, Rs. $4 / 73$ (Nold), Slg. 1974, 491.

8 EuGH, Rs. $4 / 73$ (Nold), Slg. 1974, 491, Rn. 13. Diese Formulierung fand später Eingang in Art. F EUV (Maastricht) bzw. Art. 6 EUV.

9 EuGH, Rs. 4/73 (Nold), Slg. 1974, 491, Rn. 12 f.

$10 \mathrm{Zu}$ den Entscheidungen des EuGH zum Grundrechtsschutz in der Zeit bis zur Solange-II-Entscheidung des BVerfG gehören EuGH, Rs. 44/79 (Hauer), Slg. 1979, 3727; EuGH, Rs. $59 / 83$ (Biovilac), Slg. 1984, 4057; EuGH, Rs. 250/83 (Finsider), Slg. 1985, 142; EuGH, Rs. 149/77 (Defrenne III), Slg. 1978, 1365. Zur Entwicklung des europäischen Grundrechtsschutzes zwischen den beiden Solange-Entscheidungen s. auch BVerfG, Urt. v. 22.10.1986, BVerfGE 73, 339, 378 ff. - Solange II. Zur EMRK äußerte sich der EuGH auch in den Entscheidungen EuGH, Rs. 222/84 (Johnston/The Chief Constable of the Royal Ulster Constabulary), Slg. 1986, 1651; EuGH, Rs. 36/75 (Rutili), Slg. 1975, 1219, 1232.

11 BVerfG, Urt. v. 22.10.1986, BVerfGE 73, 339, 385 - Solange II; dazu M. von Unger, "So lange" nicht mehr: Das BVerfG behauptet die normative Freiheit des deutschen Rechts, NVwZ 2005, S. 1266, 1269.

12 BVerfG, Urt. v. 22.10.1986, BVerfGE 73, 339, 387 - Solange II. Diese Beschränkung in der Zuständigkeit als Hüter des Grundrechtsschutzes in Deutschland hat das Gericht in ständiger Rechtsprechung bestätigt, s. insbesondere BVerfG, Urt. v. 7.6.2000, BVerfGE 102, 147 - Bananenmarkt; vgl. auch M. von Unger (Fn. I1).

13 BVerfG, Urt. v. 22.10.1986, BVerfGE 73, 339, 383 f. - Solange II.

14 Entschließung des Europäischen Parlamentes v. 12.4.1989 zur Erklärung der Grundrechte und Grundfreiheiten, ABI. EG 1989 C 120/51 sowie Entschließung des Europäischen Parlamentes v. 10.2.1994 zur Verfassung der Europäischen Union - Titel VIII: Von der Union verbürgte Menschenrechte, ABl. EG 1994 C 61/155, S. 166 ff.; F. Schorkopf, in: Grabitz/Hilf/Nettesheim (Hrsg.), Das Recht der Europäischen Union, 2012, Art. 6 EUV, Rn. 2.

15 Vertrag über die Europäische Union v. 7.2.1992, ABl. EG 1992 C 191/01, S. 4.

16 Charta der Grundrechte der Europäischen Union v. 18.12.2000, ABl. EG 2000 C 364/01; F. Schorkopf, in: Grabitz/Hilf/Nettesheim (Fn. 14), Art. 6 EUV, Rn. 4. 
im Dezember 2009 hat die Grundrechtecharta nun endlich auch den Rang von Primärrecht, Art. 6 Abs. 1 EUV. Darüber hinaus steht der Beitritt der EU zur EMRK bevor. ${ }^{17}$

\section{Demokratiedefizit auf europäischer Ebene}

\section{a) Mehr Rechte für das Europäische Parlament}

Ein wiederkehrendes Thema in den Entscheidungen des Bundesverfassungsgerichts ist die Rolle des Europäischen Parlaments. In der Entscheidung Solange I bemängelte das Bundesverfassungsgericht, die Europäische Wirtschaftsgemeinschaft besäße kein unmittelbar demokratisch legitimiertes, allgemein gewähltes Parlament mit Gesetzgebungsbefugnissen, dem die gesetzgebenden Gemeinschaftsorgane voll verantwortlich seien. ${ }^{18}$ Das damals noch Versammlung genannte Parlament erfülle die an eine Volksvertretung zu stellenden Anforderungen nicht. ${ }^{19}$

\section{b) Umsetzung der Vorgaben}

Nur zwei Jahre nach der Entscheidung Solange I wurde der Direktwahlakt ${ }^{20}$ verabschiedet, in dessen Folge 1979 die ersten direkten Wahlen zum Europäischen Parlament abgehalten wurden. Mit der Einheitlichen Europäischen Akte wurden dann die parlamentarischen Rechte ausgebaut und das Verfahren der Zusammenarbeit etabliert. ${ }^{21}$ Zudem wurden die Mitwirkungsrechte des Europäischen Parlamentes stetig erweitert, bis schließlich das Mitentscheidungsverfahren als ordentliches Gesetzgebungsverfahren gemäß Art. 294 AEUV installiert wurde. In der Lissabon-Entscheidung räumte das Bundesverfassungsgericht dann ein, dass die demokratische Legitimation der Union wachse, selbst wenn sie noch kein Maß erreicht habe, das dem des deutschen Staates entspreche. ${ }^{22}$

17 Art. 6 Abs. 2 S. 1 EUV; Protokoll Nr. 14 zur EMRK v. 14.5.2011.

18 BVerfG, Urt. v. 29.5.1974, BVerfGE 37, 271, 280 - Solange I.

19 BVerfG, Urt. v. 29.5.1974, BVerfGE 37, 271, 280 - Solange I.

20 Akt zur Einfuhrung allgemeiner unmittelbarer Wahlen der Abgeordneten der Versammlung v. 20.9.1976, ABl. EG 1976 L 278/1. Art. 1 DWA lautete in der ursprünglichen Fassung: „Die Abgeordneten der Völker der in der Gemeinschaft vereinigten Staaten in der Versammlung werden in allgemeiner, unmittelbarer Wahl ge-
wählt.“.

21 Vgl. Art. 6 ff. EEA v. 29.6.1987, ABl. EG 1987 L 169/1, S. 5 ff.

22 BVerfG, Urt. v. 30.6.2009, BVerfGE 123, 267, 368 ff., Rn. 272 ff. - Lissabon; vgl. dazu U. Everling, Europas Wukunft unter der Kontrolle der nationalen Verfassungsgerichte, EuR 2010, S. 91, 97, der eine ausreichende vermisst. 


\section{Kompetenzen der EU}

a) Das BVerfG als Hüter des Prinzips der begrenzten Einzelermächtigung und des Subsidiaritätsprinzips

Ein weiteres Thema der Entscheidungen des Bundesverfassungsgerichts zum europäischen Recht ist das angebliche Demokratiedefizit durch Verlagerung von Kompetenzen auf die Europäische Gemeinschaft bzw. Union. Im Maastricht-Urteil diskutierte das Bundesverfassungsgericht eingehend die Problematik einer Kompetenz-Kompetenz der Gemeinschaft und warnte mit deutlichen Worten:

"Wenn eine dynamische Erweiterung der bestehenden Verträge sich bisher auf eine großzügige Handhabung des Art. 235 EWGV im Sinne einer "Vertragsabrundungskompetenz", auf den Gedanken der inhärenten Zuständigkeiten der Europäischen Gemeinschaften ("implied powers") und auf eine Vertragsauslegung im Sinne einer größtmöglichen Ausschöpfung der Gemeinschaftsbefugnisse ("effet utile") gestützt hat (vgl. Zuleeg, in: von der Groeben/Thiesing/Ehlermann, EWG-Vertrag, 4. Aufl. 1991, Art. 2 Rn. 3), so wird in Zukunft bei der Auslegung von Befugnisnormen durch Einrichtungen und Organe der Gemeinschaften zu beachten sein, daß der Unions-Vertrag grundsätzlich zwischen der Wahrnehmung einer begrenzt eingeräumten Hoheitsbefugnis und der Vertragsänderung unterscheidet, seine Auslegung deshalb in ihrem Ergebnis nicht einer Vertragserweiterung gleichkommen darf; eine solche Auslegung von Befugnisnormen würde für Deutschland keine Bindungswirkung entfalten. "23

Zudem betonte das Bundesverfassungsgericht das Subsidiaritätsprinzip und gab der deutschen Regierung und dem Parlament konkrete Hausaufgaben auf:

.,Inwieweit das Subsidiaritätsprinzip einer Erosion mitgliedstaatlicher Zuständigkeiten und damit einer Entleerung der Aufgaben und Befugnisse des Bundestages entgegenwirken wird, hängt - neben der an das Subsidiaritätsprinzip gebundenen Rechtsprechung des Europäischen Gerichtshofs - maßgeblich von der Praxis des Rates als dem eigentlichen Gesetzgebungsorgan der Gemeinschaft ab. Hier hat die Bundesregierung ihren Einfluß zugunsten einer strikten Handhabung des Art. 3 b Abs. 2 EGV geltend zu machen und damit die ihr durch Art. 23 Abs. 1 Satz 1 GG auferlegte Verfassungspflicht zu erfüllen. Der Bundestag hat seinerseits die Möglichkeit, über sein in Art. 23 Abs. 3 GG begründetes Mitwirkungsrecht an der internen deutschen Willensbildung auf die Ratspraxis einzuwirken und sie im Sinne des Subsidiaritätsprinzips zu beeinflussen. Auch der Bundestag erfüllt damit eine ihm nach Art. 23 Abs. 1 Satz 1 GG obliegende Verfassungspflicht. Im Übrigen ist zu erwarten, daß sich auch der Bundesrat des 
Subsidiaritätsprinzips besonders annehmen wird (vgl. Goppel, Die Bedeutung des Subsidiaritätsprinzips, EuZW 1993, S. 367). “24

b) Der Kompetenzkatalog des Lissabon-Vertrags, Art. $2 f f$. AEUV

In den folgenden Jahren wurde (auch) von deutscher Seite zur Abgrenzung der Aufgaben der Union ein klarer Kompetenzkatalog gefordert. ${ }^{25}$ Seit dem Vertrag von Lissabon enthält das europäische Primärrecht mit Art. $2 \mathrm{ff}$. AEUV ausdrücklich verschiedene Kompetenzarten und -kataloge, die an die Art. 70 ff. GG erinnern. ${ }^{26}$ Der Katalog, der zwischen ausschließlichen Kompetenzen der EU und geteilten Zuständigkeiten unterscheidet, soll auch die Aufgabenverteilung zwischen EU und Mitgliedstaaten verdeutlichen und dem Prinzip der begrenzten Einzelermächtigung Rechnung tragen. ${ }^{27}$ Eine staatsanaloge Ausgestaltung der Union ist nach Ansicht des Bundesverfassungsgerichts nicht nötig, solange das Prinzip der begrenzten Einzelermächtigung gilt und die Balance zwischen den Zuständigkeiten der Union und der Mitgliedstaaten gewahrt bleibt. ${ }^{28}$

\section{Beteiligung des nationalen Parlamentes}

\section{a) Mehr Rechte für das nationale Parlament}

Um das Demokratiedefizit auf europäischer Ebene abzubauen, ist neben der stärkeren „Demokratisierung“ der Unionsorgane eine Einbindung der mitgliedstaatlichen Parlamente notwendig. ${ }^{29}$ Schon im Maastricht-Urteil formulierte das Bundesverfassungsgericht:

„Im Staatenverbund der Europäischen Union erfolgt mithin demokratische Legitimation notwendig durch die Rückkoppelung des Handelns europäischer Organe an die Parlamente der Mitgliedstaaten. "30

Im Urteil zum ESM-Vertrag hebt das Bundesverfassungsgericht hervor, dass Deutschland beim ESM durch den Finanzminister vertreten wird, der dem Bundes-

24 BVerfG, Urt. v. 12.10.1993, BVerfGE 89, 155, 210 - Maastricht.

25 M. Nettesheim, in: Grabitz/Hilf/Nettesheim (Fn. 14), Art. 2 AEUV, Rn. 7; Entschließung des Bundesrates zur Eröffnung der Regierungskonferenz zu institutionellen Fragen v. 4.2.2000, BR-Drucks. 61/00. Für einen Überblick über die Stimmen zum Kompetenzkatalog vor dessen Entstehung s. M. Bungenberg, Dynamische Integration, Art. 308 und die Forderung nach dem Kompetenzkatalog, EuR 2000, S. 879 ff. Zur Frage der Kompetenzverteilung insgesamt s. C. Calliess, Kontrolle zentraler Kompetenzausübung in Deutschland und Europa: Ein Lehrstück für die Europäische Verfassung, EuGRZ 2003, S. 181.

26 C. Calliess (Fn. 25), betrachtete die europäischen Kompetenznormen schon 2003 als ,differenzierter ausformuliert" als die deutschen.

27 M. Kotzur, in: Geiger/Khan/Kotzur (Hrsg.), EUV/AEUV, 2010, 5. Aufl., Art. 2 AEUV, Rn. 1.

28 BVerfG, Urt. v. 30.6.2009, BVerfGE 123, 267, 268 f., Rn. 272-Lissabon.

29 BVerfG, Urt. v. 30.6.2009, BVerfGE 123, 267, 381, Rn. 297 - Lissabon.

30 BVerfG, Urt. v. 12.10.1993, BVerfGE 89, 155, 185 f. - Maastricht. 
tag gegenüber rechenschaftspflichtig und mittelbar verantwortlich ist. ${ }^{31}$ So seien die Rechte des Bundestags gewahrt.

\section{b) Umsetzung auf europäischer Ebene: Mitwirkung der nationalen Parlamente, Art. 12 EUV und die Protokolle Nr. 1 und Nr. 2}

Der Lissabon-Vertrag, ${ }^{32}$ der Ende 2009 in Kraft trat, bedeutete für die nationalen Parlamente eine erhebliche Stärkung ihrer Position auf europäischer Ebene. ${ }^{33}$ Die Rolle der nationalen Parlamente innerhalb der Rechtsetzung der Union ist primärrechtlich in Art. 12 EUV geregelt, der den Volksvertretungen der Mitgliedstaaten weitreichende Unterrichtungs-, Überwachungs- und Beteiligungsrechte gewährt. Nähere Ausgestaltung erfahren die Rechte in zwei Protokollen zu den Verträgen. Das erste Protokoll ${ }^{34}$ betrifft die Rolle der nationalen Parlamente in der Union. Dem Vertrag von Lissabon wurde außerdem als Protokoll Nr. 2 das Protokoll über die Anwendung der Grundsätze der Subsidiarität und der Verhältnismäßigkeit ${ }^{35}$ als Ergänzung zu Art. 5 EUV angefügt. Dieses Subsidiaritätsprotokoll ermöglicht es den nationalen Parlamenten unter anderem, die Wahrung des Subsidiaritätsgrundsatzes vor dem EuGH einzuklagen (Art. 8) ${ }^{36}$ und gegebenenfalls einen Gesetzgebungsakt zu überarbeiten (Art. 7 Abs. 2).

\section{Verbleibende ausschließliche Kompetenzen der Mitgliedstaaten}

\section{a) „Ewige" Kompetenzen der Nationalstaaten}

Während das Bundesverfassungsgericht in der Maastricht-Entscheidung noch hervorhob, dass der EU keine Kompetenz-Kompetenz zustehe, ${ }^{37}$ betonte es in der Lissabon-Entscheidung quasi spiegelbildlich, inwieweit den nationalen Parlamenten ausschließliche Kompetenzen verbleiben müssten. Leitsatz 3 lautete:

„Die europäische Vereinigung auf der Grundlage einer Vertragsunion souveräner Staaten darf nicht so verwirklicht werden, dass in den Mitgliedstaaten kein ausreichender Raum zur politischen Gestaltung der wirtschaftlichen, kulturellen und sozialen Lebensverhältnisse mehr bleibt. Dies gilt insbesondere für Sachbereiche, die die Lebensumstände der Bürger, vor allem ihren von den Grundrech-

31 BVerfG, Urt. v. 12.9.2012 = NJW 2012, 3145, 3157, Rn. 289-ESM.

32 Vertrag von Lissabon zur Änderung des Vertrages über die Europäische Union und des Vertrages zur Gründung der Europäischen Gemeinschaft v. 17.12.2007, ABl. EU 2007 C 306/2, in Kraft getreten am 1.12.2009.

33 Ausführlich E. Brok/M. Selmayr, Der Vertrag der Parlamente als Gefahr für die Demokratie? Zu den offensichtlich unbegründeten Verfassungsklagen gegen den Vertrag von Lissabon, integration 2008, S. 217, $225 \mathrm{ff}$.

34 Protokoll Nr. 1 über die Rolle der nationalen Parlamente in der Europäischen Union v. 13.12.2007, ABl. EU 2007 C 306/148; konsolidierte Fassung v. 30.3.2010, AB1. EU 2010 C 83/203.

35 Protokoll Nr. 2, das Protokoll über die Anwendung der Grundsätze der Subsidiarität und der Verhältnismäßigkeit v. 13.12.2007, ABl. EU 2007 C 306/150; konsolidierte Fassung v. 30.3.2010, ABl. EU 2010 C 83/206.

36 Zu den Mechanismen des Subsidiaritätsprinzips s. R. Streinz, in: Streinz (Hrsg.), EUV/AEUV, 2012, 2. Aufl., Art. 5 EUV, Rn. $32 \mathrm{ff}$.

37 S. oben II.3.a). 
ten geschützten privaten Raum der Eigenverantwortung und der persönlichen und sozialen Sicherheit prägen, sowie für solche politischen Entscheidungen, die in besonderer Weise auf kulturelle, historische und sprachliche Vorverständnisse angewiesen sind, und die sich im parteipolitisch und parlamentarisch organisierten Raum einer politischen Öffentlichkeit diskursiv entfalten. “38

Im Einzelnen führt das Bundesverfassungsgericht dann aus:

„Als besonders sensibel für die demokratische Selbstgestaltungsfähigkeit eines Verfassungsstaates gelten seit jeher Entscheidungen über das materielle und formelle Strafrecht (1), die Verfügung über das Gewaltmonopol polizeilich nach innen und militärisch nach außen (2), die fiskalischen Grundentscheidungen über Einnahmen und - gerade auch sozialpolitisch motivierte - Ausgaben der öffentlichen Hand (3), die sozialstaatliche Gestaltung von Lebensverhältnissen (4) sowie kulturell besonders bedeutsame Entscheidungen etwa im Familienrecht, Schul- und Bildungssystem oder über den Umgang mit religiösen Gemeinschaften (5). "39

$\mathrm{Zu}$ den „ewigen“ Kompetenzen der Nationalstaaten gehöre das Budgetrecht des Parlaments. Hierzu führt das Bundesverfassungsgericht aus:

„Eine notwendige Bedingung für die Sicherung politischer Freiräume im Sinne des Identitätskerns der Verfassung (Art. 20 Abs. 1 und Abs. 2, Art. 79 Abs. 3 GG) besteht darin, dass der Haushaltsgesetzgeber seine Entscheidungen über Einnahmen und Ausgaben frei von Fremdbestimmung seitens der Organe und anderer Mitgliedstaaten der Europäischen Union trifft und dauerhaft „,Herr seiner Entschlüsse" bleibt. "40

Trotz des supranationalen Charakters der Union ,müssen die gewählten Abgeordneten des Deutschen Bundestages als Repräsentanten des Volkes die Kontrolle über grundlegende haushaltspolitische Entscheidungen behalten."41 Dem Demokratieprinzip sei nur Genüge getan, wenn ,die effektive Wahrnehmung der [national-] parlamentarischen Haushalts- und Integrationsverantwortung in jedem Fall sichergestellt ist.“"42

Um die nationale Budgethoheit auch im Zusammenhang mit der Eurokrise zu gewährleisten, stellte das Bundesverfassungsgericht in der Eilentscheidung zum ESM-Vertrag Bedingungen an dessen Wirksamkeit: Eine Bindung Deutschlands durch den ESM-Vertrag ${ }^{43}$ dürfe nur bestehen, wenn keine Vorschrift des Vertrages so ausgelegt werden könne, dass für die Bundesrepublik Deutschland ohne Zustimmung des deutschen Vertreters höhere Zahlungsverpflichtungen begründet wer-

39 , 30.6 .2009 , BVerfGE 123, 267, Ls. 3 - Lissabon.

BVerfG, Urt. v. 30.6.2009, BVerfGE 123, 267, 359, Rn. 252 - Lissabon

40 BVerfG, Urt. v. 12.9.2012, NJW 2012, 3145, 3148, Rn. 213 - ESM.

41 BVerfG, Urt. v. 12.9.2012, NJW 2012, 3145, 3148, Rn. 211 - ESM.

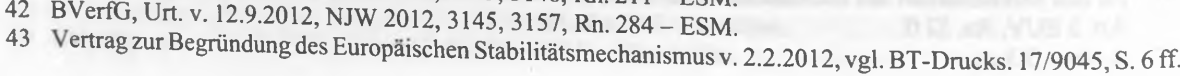


den. ${ }^{44}$ Zudem dürfe die Geheimhaltungspflicht der Beteiligten das Unterrichtungsrecht von Bundestag und Bundestag nicht torpedieren. 45

\section{b) Umsetzung durch die Mitgliedstaaten}

Das Bundesverfassungsgericht verpflichtete die Bundesregierung, diese Auslegung qua völkerrechtlichen Vorbehalts sicherzustellen. ${ }^{46}$ Der ESM-Vertrag trat mit einer entsprechenden Erklärung in Kraft. ${ }^{47}$

Dies wird auch europarechtlich nicht ohne Folgen bleiben: Indem das Bundesverfassungsgericht die Bundesregierung zum Vorbehalt verpflichtet, ${ }^{48}$ schreibt es indirekt dem EuGH die gewünschte Auslegung des ESM vor. Gemäß Art. 37 Abs. 3 ESM-Vertrag ist aber der EuGH für Streitigkeiten im Rahmen des Vertrags zuständig. Im Verfahren Pringle/Government of Ireland ${ }^{49}$ hatte der EuGH über die Zulässigkeit des ESM zu befinden. Wenig überraschend billigte der EuGH den ESM und konnte aufgrund der Vorlagefragen vermeiden, auf die jedenfalls implizite Kritik des Bundesverfassungsgerichts einzugehen. ${ }^{50}$ Es ist auch in Zukunft kaum zu erwarten, dass der EuGH den Vertrag auf die Art und Weise auslegt, die Deutschland in den völkerrechtlichen Vorbehalten ausschließt, denn weder der EuGH noch ein anderes EU-Organ kann ein Interesse daran haben, dass Deutschland aus der Eurorettung aussteigt. So haben denn auch die anderen Vertragsstaaten bekräftigt, die Auslegung des deutschen Gerichts zu teilen. ${ }^{51}$

\section{Fragen der Methodik}

\section{Formal Juristische Zurückhaltung}

\section{a) Judicial Self-Restraint}

Die Zurückhaltung, die das Bundesverfassungsgericht übt, wenn es die verschiedenen Verträge auf europäischer Ebene trotz aller Bedenken im Ergebnis für rechtmäßig erachtet, lässt methodisch an verschiedene Rechtsfiguren denken. Aus dem US-amerikanischen Recht ${ }^{52}$ stammt der Gedanke des „Judicial Self-Restraint“. Er bedeutet, dass sich die Gerichte bei ihren Entscheidungen zurückhalten und damit

47 H. Kafsack, Eurostaaten setzen ESM in Kraft, FAZ v. 9.10.2012, S. 1 f.

48 Zur Notwendigkeit der Vorbehalte unter Androhung der Unverbindlichkeit BVerfG, Urt. v. 12.9.2012, NJW 2012, 3145, 3153, Rn. 253, 259 - ESM.

49 EuGH, Rs. C-370/12 (Thomas Pringle/Government of Ireland, Ireland, The Attorney General), WM $2012,2364$.

(Thomas Pringle/Government of Ireland, Ireland, The Attorney General), WM 2012, 2364.

51 Für den Wortlaut der Erklärung s. BT-Drucks. 17/10767 v. 25.9.2012, S. 5. S. auch W. Mussler, Eurogruppe erfüllt Karlsruher Bedingung, FAZ v. 15.9.2012, S. 1.

52 US Supreme Court, Urt. v. 6.1.1936, United States v. Butler, 297 U.S. 1, 79 (1935); sehr anschaulich Cox, 47 (1987); Posner, 100 Cal.L.Rev. 519 (2012); Ribble, 26 Virg.L.Rev. 981,984 (1940). 
dem demokratischen legitimierten Gesetzgeber letztlich in wichtigen politischen Fragestellungen ein Ermessen einräumen, so dass die Klage im Zweifel abzuweisen ist. ${ }^{53}$ Den Einschätzungsspielraum des Gesetzgebers betont auch das Bundesverfassungsgericht, indem es etwa formuliert, „der Gesetzgeber [verfüge] namentlich mit Blick auf die Frage der Eintrittsrisiken und die zu erwartenden Folgen für die Handlungsfreiheit des Haushaltsgesetzgebers über einen weiten Einschätzungsspielraum, den das Bundesverfassungsgericht grundsätzlich zu respektieren hat. " 54 Bezeichnenderweise hat etwa Peter Gauweiler schon oft vor dem Bundesverfassungsgericht eine Kompetenzüberschreitung moniert, im Ergebnis bislang aber noch keine Klage gewonnen.

\section{b) Normative Kraft des Faktischen}

Methodisch interessant ist noch eine zweite Rechtsfigur, die Georg Jellinek schon vor mehr als 100 Jahren entwickelte: die „normative Kraft des Faktischen“. 55 Zwar wissen wir spätestens seit Hans Kelsen, dass zwischen dem faktischen „Sein“ und dem normativen „Sollen“ streng zu trennen ist. ${ }^{56}$ Tatsächlich wirkt aber nicht selten doch das „Sein“ auf das „Sollen“.

Im Rahmen von inzwischen 28 Mitgliedstaaten drängt sich diese Rechtsfigur geradezu auf. Ein einzelnes Verfassungsgericht würde die Fakten verkennen, wolle es europäisches Recht im Alleingang für unverbindlich erklären. ${ }^{57}$ Politisch hatte das BVerfG keine andere Wahl als die europäischen Änderungen - wenngleich eventuell widerstrebend - zu billigen. ${ }^{58}$ Selbst die Beschwerdeführer erwarteten in vielen Fällen kein „Nein“ zum Änderungsvertrag. ${ }^{59}$ Gerade die vermeintliche Verfassungswidrigkeit des Maastricht- oder des Lissabon-Vertrages oder noch drastischer jetzt die Vereinbarkeit des ESM-Vertrags mit dem Grundgesetz zeigen sehr deutlich, dass eine Entscheidung des Bundesverfassungsgerichts nicht nur Auswirkungen in den Mitgliedsstaaten der EU hat, sondern weltweite Relevanz haben kann:

53 BVerfG, Urt. v. 31.7.1973, BVerfGE 36, 1, 14 f. und Ls. 2 - Grundlagenvertrag; abweichende Meinung der Richter Rupp-van Brünneck und Simon zu BVerfG, Urt. v. 25.2.1975, BVerfGE 39, 169 f. - Schwangerschaftsabbruch; für die einstweilige Anordnung s. BVerfG, Urt. v. 10.12.1953, BVerfGE 3, 52, 55 - Weihnachtsgeld; C. Rau, Selbst entwickelte Grenzen in der Rechtsprechung des United States Supreme Court und des Bundesverfassungsgerichts, 1996; H. Rupp, Some Remarks on Judicial Self-Restraint, 21 Ohio St.L.J. 503 (1960); G. Schuppert, Self-restraints der Rechtsprechung. Überlegungen zur Kontrolldichte in der Verfassungs- und Verwaltungsgerichtsbarkeit, DVBI. 1988, S. $1191 \mathrm{ff}$.

54 S. etwa BVerfG, Urt. v. 12.9.2012, NJW 2012, 3145, 3149, Rn. 217 - ESM.

55 G. Jellinek, Allgemeine Staatslehre, 3. Aufl. 1914, S. 338.

56 H. Kelsen, Reine Rechtslehre, 2. Aufl. 1960, S. 5; vorher schon ders., Eine Grundlegung der Rechtssoziologie, Archiv für Sozialwissenschaft und Sozialpolitik 1915, S. 839, 841. 57 So erkennt das BVerfG selbst, „,wenn jeder Mitgliedstaat für sich in Anspruch nähme, durch eigene Gerichte terlaufen werden, und die einheitliche Anwendung des Unionsrechts wäre gefährdet.", BVerfG, Urt. v. 6.7.2010, BVerfGE 126, 286, 303, Rn. 57 - Honeywell.

58 So zum Lissabon-Urteil C. Calliess, Das Ringen des Zweiten Senats mit der Europäischen Union: Über das Ziel hinausgeschossen..., ZEuS 2009, S. 559, 564, 582. Dabei sollte nicht vergessen werden, dass die Zustimmungs-

59 Zur Verfassungsbeschwerderträgen von politischen Mehrheiten in Bundestag und Bundesrat getragen wurden tricht's Epigones At Sea, GLJ 2009, 1207. 

Als das Urteil des BVerfG zum ESM erging, stiegen die Kurse an den Märkten
deutlich an. ${ }^{60}$

\section{2. „Judicial Activism" und das BVerfG als europäischer Gesetzgeber}

\section{a) Appellentscheidungen mit Nachbesserungsvorbehalt}

Und doch ist das nur eine Blickrichtung: Zwar mag es auf den ersten Blick scheinen, als habe das Bundesverfassungsgericht europäische Änderungen wie den Maastricht- oder Neuerungen wie den ESM-Vertrag lediglich abgenickt. Von einer generellen Zurückhaltung des Bundesverfassungsgerichts, gar von einer anstandslosen Akzeptanz dessen, was auf europäischer Ebene geschieht, kann jedoch nicht die Rede sein. Dem "Ja" des Gerichts folgte regelmäßig ein großes „Aber“, mit dem es seine Bedenken und Vorbehalte zum Ausdruck brachte und der Europäischen Union den angeblich einzig noch verfassungsmäßigen Weg aufzeigte.

Im deutschen Verfassungsrecht werden solche Entscheidungen als „Appellentscheidung mit Nachbesserungsvorbehalt" ${ }^{“ 61}$, „Noch-Verfassungsmäßigkeitserklärung"62 oder Entscheidung des „Weiterbeobachten(s) und gegebenenfalls (N)achbessern(s)" 63 bezeichnet. Sie ermöglichen dem Bundesverfassungsgericht die Rücksichtnahme auf etwaige unerwünschte politische Folgen einer Nichtigerklärung der Norm ${ }^{64}$ und sind zahlreich bekannt, wie etwa im Bereich des Steuerrechts das Urteil zur Vermögenssteuer. ${ }^{65}$

\section{b) Die Drohung mit der fehlenden Bindungswirkung und die Ultra vires-Kontrolle}

Eine europäische Norm kann das Bundesverfassungsgericht nicht für nichtig erklären; lediglich für das Zustimmungsgesetz bleibt diese Möglichkeit. Allerdings soll für europäische Rechtsakte, die gegen das Grundgesetz verstoßen, der Vorrang des Europarechts nach Ansicht des Bundesverfassungsgerichts nicht gelten. Es droht für diese - bislang hypothetischen - Fälle regelmäßig damit, Deutschland sei dann nicht an das betreffende europäische Recht gebunden, etwa mit den Worten:

60 S. oben Fn. 1.

61 P. Häberle, Die Verfassungsbeschwerde im System der bundesdeutschen Verfassungsgerichtsbarkeit, JöR n.F. 1997, S. 89, 126, nennt als Urheber des Begriffs „Appellentscheidung“ die Verfassungsrichterin Rupp-von Brünneck. S. auch T.-H. Yang, Die Appellentscheidungen des Bundesverfassungsgerichts, 2003.

62 H. Bethge, in: Maunz/Schmidt-Bleibtreu/Klein/Bethge (Hrsg.), Bundesverfassungsgerichtsgesetz, 2012, $\$ 31$, Rn. 181, 250.

63 H. Bethge, in: Maunz/Schmidt-Bleibtreu/Klein/Bethge (Fn. 62), §31, Rn. 251; s. dazu P. Badura, Die verfassungsrechtliche Pflicht des gesetzgebenden Parlaments zur, Nachbesserung“ von Gesetzen, in: FS Eichenberger, 1982, S. 481.

64 K. Schlaich/S. Korioth, Das Bundesverfassungsgericht, 2012, 9. Aufl., Rn. 439.

65 BVerfG, Urt. v. 22.6.1995, BVerfGE 93, 121 - Vermögenssteuer; s. auch BVerfG, Urt. v. 8.8.1978, BVerfGE 49, 89 - Atomgesetz; BVerfG, Urt. v. 16.11.1992, BVerfGE 87, 348 - Versorgungsausgleich. Diese Urteile sind nicht $\mathrm{zu}$ verwechseln mit jenen, in denen das BVerfG ein Gesetz für nichtig erklärt, seine Wirksamkeit aber zeitlich ausdehnt, um dem Gesetzgeber die Möglichkeit der Reaktion zu geben (z.B. BVerfG, Urt. v. 9.2. 2010, $1 \mathrm{BvL} 1 / 09$ - Hartz IV-Regelsätze). Vgl. P. Badura (Fn. 63); T.-H. Yang (Fn. 61). 
„[...] so wird in Zukunft bei der Auslegung von Befugnisnormen durch Organe und Einrichtungen der Gemeinschaften zu beachten sein, dass [die] Auslegung [des EU-Vertrags] deshalb in ihrem Ergebnis nicht einer Vertragserweiterung gleichkommen darf; eine solche Auslegung von Befugnisnormen würde für Deutschland keine Bindungswirkung entfalten. "66

Darüber hinaus behält sich das Bundesverfassungsgericht vor, selbst zu prüfen, ob die EU oder der EuGH die eigenen Kompetenzen überschreiten. Da die Kompetenzen durch nationales Recht übertragen werden, obliege diese Prüfung dem Bundesverfassungsgericht. Im Übrigen müsse das BVerfG etwaige Kompetenzüberschreitungen schon deshalb kontrollieren, weil der EuGH sonst tatsächlich eine Kompetenz-Kompetenz hätte und nicht kontrolliert würde. ${ }^{67}$

Auch die (Verfassungs-)Gerichte zahlreicher anderer Mitgliedstaaten betonen, dass bestimmte Inhalte ihrer nationalen Verfassungen durch das Europarecht nicht geändert werden können und erkennen den Vorrang des Europarechts insoweit nicht an. ${ }^{68}$ Der dänische Oberste Gerichtshof etwa begründete in seiner Maastricht-Entscheidung ${ }^{69}$ aber eine Vermutung zugunsten der Rechtsauffassung des EuGH. ${ }^{70} \mathrm{Im}$ Einzelnen verwies das Gericht auf das Prinzip der begrenzten Einzelermächtigung, dem Rechnung getragen werde, ${ }^{71}$ und das Einstimmigkeitsprinzip im Rat (Art. 235 EGV-Maastricht), das der dänischen Regierung ein Veto einräumte. ${ }^{72}$

\section{c) Judicial Activism}

Nicht nur mit den „Ja, aber“-Vorgaben, sondern spätestens mit der Drohung der fehlenden Bindungswirkung europäischer Rechtsakte ist ganz offensichtlich der Bereich juristischer Zurückhaltung, des ,judicial-self-restraint", verlassen. Vielmehr wird ganz offensichtlich eine Rechtspolitik betrieben, die in den USA als liberaler ,judicial activism“ beschrieben wird. ${ }^{73}$ In Deutschland kritisierte ein Minderheitsvotum bereits 1975, dass das Bundesverfassungsgericht in diesen Fällen seine Kompetenzen überschreite:

66 BVerfG, Urt. v. 12.10.1993, BVerfGE 89, 155, 210 - Maastricht. S. auch BVerfG, Urt. v. 30.6.2009, BVerfGE 123, 267, 353 Rn. 240 - Lissabon.

67 M. Gerhardt, Europa als Rechtsgemeinschaft: Der Beitrag des Bundesverfassungsgerichts, ZRP 2010, S. 161, 163.

68 S. dazu die sehr gute Übersicht bei F. Mayer, in: Grabitz/Hilf/Nettesheim (Fn. 14), Art. 19 EUV, Rn. 92 ff. Ähnlich wie das Bundesverfassungsgericht urteilt der polnische Verfassungsgerichtshof: Trybunal Konstytucyjny, Urt. v. 11.5.2005, AZ.K 18/04, in deutscher Übersetzung EuR 2006, S. 236, 243.

69 Dänischer Oberster Gerichtshof, Urt. v. 6.4.1998, UfR 1998, S. 800 ff.; auszugsweise deutsche Übersetzung s. F. Thomas, Das Maastricht-Urteil des dänischen Obersten Gerichtshofs vom 6. April 1998, ZaöRV 1998, S.
$879,901 \mathrm{ff}$.

70 F. Thomas (Fn. 69), S. 905 f.

71 F. Thomas (Fn. 69), S. 903.

72 F. Thomas (Fn. 69), S. 904.

73 US Supreme Court, Urt. v. 8.4.1895, Pollock v. Farmers' Loan \& Trust Co., 157 U.S. 429 (1895); US Supreme Court, Urt. v. 9.4.1923, Adkins v. Children's Hosp. 261 U.S. 525 (1923); US Supreme Court, Urt. v. 28.5.1928, Ribnick v. McBride, 277 U.S. 350 (1928); Cox, 47 Md.L.Rev. 118, 121 ff. (1987). 
„Das Gebot richterlicher Selbstbeschränkung (judicial self-restraint), das als das "Lebenselexier" (sic!) der Rechtsprechung des Bundesverfassungsgerichts bezeichnet worden ist, gilt vor allem, wenn es sich nicht um die Abwehr von Übergriffen der staatlichen Gewalt handelt, sondern wenn dem vom Volk unmittelbar legitimierten Gesetzgeber im Wege der verfassungsgerichtlichen Kontrolle Vorschriften für die positive Gestaltung der Sozialordnung gemacht werden sollen. Hier darf das Bundesverfassungsgericht nicht der Versuchung erliegen, selbst die Funktion des zu kontrollierenden Organs zu übernehmen, soll nicht auf lange Sicht die Stellung der Verfassungsgerichtsbarkeit gefährdet werden. "74

Das Bundesverfassungsgericht gibt in seiner Rechtsprechung eine klare Linie vor, der der europäische Gesetzgeber folgt, wenn er etwa nach der Bemängelung des Grundrechtsstandards diesen anhebt. Die weitreichenden Entscheidungen des Bundesverfassungsgerichts sind also aus zwei Gründen kritisch unter die Lupe zu nehmen: Zum einen stellt sich - auch schon auf nationaler Ebene - die Frage, inwieweit ein demokratisch nicht legitimiertes Organ wie das Bundesverfassungsgericht quasi-gesetzgeberische Entscheidungen treffen darf, zumal im Anschluss an eine Mehrheitsentscheidung im Parlament und nicht immer unter Beachtung des - wenngleich vielzitierten ${ }^{75}$ - Ermessensspielraums des Gesetzgebers. Zum anderen gewinnt diese Frage im europäischen Zusammenhang zusätzlich an Brisanz: Darf das Gericht eines Mitgliedstaats in derartig großem Maße die Politik und das Recht der Union und damit auch aller anderen 27 Mitgliedstaaten bestimmen?

\section{Inhaltliche Parameter zulässiger Rechtsfortbildung}

\section{a) Angemessenheit des Ergebnisses, Schutzbedürftigkeit, Akzeptanz}

Streitigkeiten zu den juristischen Methodenfragen werden in Deutschland inzwischen auch vor dem Bundesverfassungsgericht ausgetragen, etwa im Rahmen der Entscheidung zur Rügeverkümmerung. ${ }^{76}$ Das Bundesverfassungsgericht stellt Kriterien zur Prüfung der Zulässigkeit von Rechtsfortbildung auf. Es muss sich also gefallen lassen, dass auch seine eigenen Entscheidungen, einschließlich der europarechtlichen, insoweit untersucht werden.

Minderheitsvotum Rupp-von Brünneck/Simon zu BVerfG, Urt. v. 25.2.1975, BVerfGE 39, 1, 68, 69 f. - Schwangerschaftsabbruch; kritisch auch etwa die Minderheitsvoten in Fn. 76.

75 So zuletzt BVerfG, Urt. v. 12.9.2012, NJW 2012, 3145, 3149, Rn. 217 - ESM.

76 Das Sondervotum der Richter Voßkuhle, Osterloh und Di Fabio brandmarkte die Mehrheitsentscheidung dabei als unzulässige Rechtsfortbildung: BVerfG, Beschl. v. 15.1.2009, BVerfGE 122, 248, 282 ff., Rn. 95 ff. - Rügeverkümmerung. Hierzu C. Möllers, Nachvollzug ohne Maßstabbildung: Richterliche Rechtsfortbildung in der Rechtsprechung des Bundesverfassungsgerichts, JZ 2009, S. 668. Vorher schon BVerfG, Urt. v. 11.10.1979, BVerfGE 49, 304, 321 - Sachverständigenhaftung; BVerfG, Úrt. v. 19.10.1983, BVerfGE 65, 182, Sozialplan. 
Jüngst wurden Parameter präzisiert, die „, für“ oder ,gegen“ eine Rechtsfortbildung streiten. ${ }^{77}$ Die Angemessenheit des Ergebnisses und vor allem der Rechtsfolge ist Voraussetzung für die Lückenfüllung und Analogiebildung. ${ }^{78} \mathrm{Da}$ der Rechtsanwender bei der Fortbildung des Rechts ,Neuland“ betritt, hat er, ähnlich wie der Gesetzgeber bei der Schaffung von Gesetzen, die Rechtsfolgen seines Tuns zu berücksichtigen. ${ }^{79}$ Das deckt sich mit den Überlegungen von Hassemer: Die juristische Methodenlehre dient nur der Begründung und Darstellung der Entscheidung, sie ist aber nicht als solche für die Rechtsfindung heranzuziehen. Auslöser einer Rechtsfortbildung ist oft die Frage, ob ein bestehender Missstand durch Rechtsfortbildung abgestellt werden kann. Hier kommen Wertungen und Gerechtigkeitsvorstellungen zum Tragen. Das Rechtsverweigerungsverbot kann als Begründung herangezogen werden. Es ist insoweit nicht nur Formalargument, sondern erinnert eher an die Radbruch'sche Formel des ,unerträglichen Unrechts“.80 Umgekehrt drängt sich die Lückenfüllung nicht auf, wenn dem Kläger auch anderweitig angemessener Rechtschutz ermöglicht wird. Dann erscheint ein Kompetenzzuwachs der Gerichtsbarkeit zur Rechtsfortbildung aufgrund fehlenden Rechtsschutzbedürfnisses nicht mehr gerechtfertigt. Die praktischen Konsequenzen einer durch eine Rechtsnorm angeordneten Rechtsfolge sind oft der entscheidende Gesichtspunkt, um eine Analogie zu bejahen oder zu verneinen. ${ }^{81}$ Damit kann einer folgenorientierten Auslegung eine ausschlaggebende Rolle zukommen.

In der Rechtsliteratur wird nur vereinzelt gefordert, die überwiegende Akzeptanz einer Rechtsansicht als Kriterium zur Rechtsfortbildung heranzuziehen. ${ }^{82}$ Die Rechtsprechung ist hier deutlicher, wenn sie auf die herrschende Ansicht abstellt, etwa dass der Deutsche Juristentag eine bestimmte Rechtsansicht beschlossen hat. Zudem werden rechtsvergleichende Entscheidungen herangezogen, die für die gewünschte Rechtsfortbildung sprechen. Dies gilt insbesondere dann, wenn in zahlreichen anderen Staaten ein Regelungskonzept besteht, während (nur) in Deutschland eine Gesetzeslücke vorliegt. Schließlich ist zu erörtern, ob die Rechtsfortbil-

77 T. Möllers, Ein Vier-Stufen-System zur Rationalisierung der Grenze zulässiger Rechtsfortbildung, in: Stelmach/ Schmidt (Hrsg.), Krakauer-Augsburger Rechtsstudien, 2011, S. 109, 121 ff. = ders., Ein Vierstufen-System zur Rationalisierung der Grenze zulässiger Rechtsfortbildung, in: FS Roth, 2011, S. 473, $481 \mathrm{ff}$.

78 R. Hemke, Methodik der Analogiebildung im öffentlichen Recht, 2006, S. 371: „So würde niemand auf die Idee kommen, eine Vorschrift analog anzuwenden, deren Rechtsfolge er nicht im ungeregelten Fall für angemessen und passend hält".

79 Sehr deutlich Art. 1 Abs. 2 schweizerisches ZGB: „Kann dem Gesetze keine Vorschrift entnommen werden, so soll der Richter nach Gewohnheitsrecht und, wo auch ein solches fehlt, nach der Regel entscheiden, die er als Gesetzgeber aufstellen würde.“.

80 Zur Radbruch'schen Formel s. G. Radbruch, Gesetzliches Unrecht und übergesetzliches Recht, SJZ 1947, S. 105, 107: „Der Konflikt zwischen Gerechtigkeit und der Rechtssicherheit dürfte dahin zu lösen sein, daß das positive, durch Satzung und Macht gesicherte Recht, auch dann den Vorrang hat, wenn es inhaltlich ungerecht und unzweckmäßig ist, es sei denn, daß der Widerspruch des positiven Rechts zur Gerechtigkeit ein solch unverträgliches Maß erreicht, dass das Gesetz als ,unrichtiges Recht“ zu weichen hat.“ Vorher ähnlich schon H. Reichel, Gesetz und Richterspruch, 1915, S. 142; s. RG, Urt. v. 18.11.1923, RGZ 107, 78.

81 G. Roth/C. Schubert, in: Säcker/Rixecker (Hrsg.), Münchener Kommentar zum BGB, 6. Aufl. 2012, § 242, Rn. 34.

82 Etwa B. Heusinger, Rechtsfindung und Rechtsfortbildung im Spiegel richterlicher Erfahrung, 1975, S. 89 f.; E. Kramer, Juristische Methodenlehre, 3. Aufl. 2010, S. 247; G. Müller, Richterliche Rechtsfortbildung im Arbeitsrecht, JuS 1980, S. 627, 635; R. Wank, Grenzen richterlicher Rechtsfortbildung, 1978, S. 42 f. 
dung ein Mehr an Rechtssicherheit erzeugt ${ }^{83}$ oder ob umgekehrt die geplante Rechtsfortbildung nicht praktikabel ist. Die Gerichte müssen bei der Frage, ob sie eine Regelung durch richterliche Rechtsfortbildung (weiter)entwickeln, zuvor abwägen, ob sie „um der Verwirklichung materialer Gerechtigkeit willen“”,die Rechtsicherheit in gewissem Umfang und für gewisse Zeit“84 einschränken oder die Schaffung gesetzlicher Regeln dem Gesetzgeber überlassen sollen. ${ }^{85}$

\section{b) Grundrechte}

Staatliche Regelungen können privatrechtliche Beziehungen zu dreipoligen Verhältnissen machen. Dem grundrechtlichen Schutzanspruch des Einen steht dann der grundrechtliche Eingriffsabwehranspruch des anderen gegenüber: „Schutz durch Eingriff" ${ }^{86}$ Grundrechte eines Betroffenen können folglich auch die Grundrechte Dritter tangieren. Diese genießen nicht per se Vorrang, sondern fließen nur als Abwägungselemente in die Prüfung ein. Je erheblicher die Rechtsgüter Dritter sind, desto eher können diese verfassungsrechtlich geschützten Rechtsgüter gegen eine Rechtsfortbildung sprechen. Plastisch ausgedrückt wird man einen Verstoß gegen die Verfassung bejahen können, wenn auch ein gleichlautendes Gesetz nicht mehr mit der Verfassung vereinbar wäre. ${ }^{87}$

\section{c) Das Kooperationsverhältnis von Rechtsprechung und Parlament}

Gerichte entwickeln vor allem dann Rechtslösungen, wenn sich der Gesetzgeber hierzu außerstande sieht. ${ }^{88}$ Nicht von ungefähr wartet der Gesetzgeber oft ab, ob die Rechtsprechung bestimmte Rechtslösungen anbietet. Hält der Gesetzgeber diese für gelungen, wird er sie in eine Gesetzesnorm überführen. ${ }^{89}$ Hält der Gesetzgeber die Rechtslösung dagegen für nicht sinnvoll, kann er ihr mit einem Gesetz entgegentreten. ${ }^{90} \mathrm{Im}$ Steuerrecht beispielsweise gehören Nichtanwendungserlasse zum Alltagsgeschäft.

83 So R. Dworkin, Law's Empire, 1986, S. 37: „Sometimes certainty of the law is more important than what the law is; this is true for rules of the road, for example, and perhaps for rules defining rights and obligations under negotiable commercial paper. But it is not always true.".

84 BVerfG, Urt. v. 18.12.1953, BVerfGE 3, 225, 237 f. - Gleichberechtigung.

85 So R. Wank (Fn. 82), S. 204.

86 C. Hillgruber, Richterliche Rechtsfortbildung als Verfassungsproblem, JZ 1996, S. 118; S. Oeter, „Drittwirkung" der Grundrechte und die Autonomie des Privatrechts, AöR 1994, S. 529, 558, 562; BVerfG, Urt. v. 2.1.1998, BVerfGE 97, 169, 175- $\$ 23$ Abs. 1 S. 2 KSchG.

87 BVerfG, Urt. v. 26.6.1991, BVerfGE 84, 212, 228 f. - Arbeitskampfrecht; S. Vogenauer, Die Auslegung von Gesetzen in England und auf dem Kontinent, 2001, S. 149. Vorher schon BVerfG, Urt. v. 11.10.1979, BVertGE $84,212,228 \mathrm{f}$. - Sachverständigenhaftung.

88 Beispiele bilden etwa die Entwicklung des Streikrechts oder des allgemeinen Persönlichkeitsrechts, ohne dass der Gesetzgeber bisher hier eine gesetzliche Regelung gefunden hat.

89 So hat er etwa die von der Rechtsprechung entwickelten Rechtsinstitute wie culpa in contrahendo oder Wegfall der Geschäftsgrundlage durch die Schuldrechtsreform erstmals im BGB normiert.

90 So für die Entwicklung des europäischen Staatshaftungsanspruchs schon T. Möllers, Doppelte Rechtsfortbildung contra legem?, EuR 1998, S. 20, 32 f. 
Diese Überlegungen lassen sich schließlich auch umdrehen: Gerade bei Risikoentscheidungen mit ungewisser Perspektive sind Verwaltung oder Gesetzgeber dem Rechtsfindungsverfahren der Gerichte oft überlegen: Den Gerichten fehlen häufig das notwendige Instrumentarium und der umfassende Überblick, um außerrechtliche Überlegungen anstellen und einbeziehen zu können; ${ }^{91}$ sie können gerade keine abstrakt-generellen Regeln aufstellen, sondern müssen konkret auf den Fall bezogen entscheiden. ${ }^{92}$

\section{d) Bedeutung für die analysierten Urteile}

Im Folgenden soll der Versuch gemacht werden, die soeben dargestellten Kriterien auf die oben genannten vier Fallgruppen zu subsumieren.

Es fällt auf, dass die Solange-I-Entscheidung die meisten Parameter erfüllt: Die fehlenden Grundrechte hinterließen eine Lücke, die einen Missstand erzeugten, der anderweitig nicht zu beheben war. Weil der (europäische) Gesetzgeber nicht handelte, sahen sich die Gerichte aufgerufen, durch ihre Rechtsprechung die erforderliche Rechtssicherheit zu erzeugen. Dass aber gerade das Bundesverfassungsgericht tätig werden musste, darf bezweifelt werden, weil ja kurz zuvor der für solche europarechtlichen Fragen zuständige EuGH mit der Nold-Entscheidung begonnen hatte, europäische Grundrechte zu entwickeln. ${ }^{93}$

Betrachtet man des Weiteren die Rechtsprechung zur Rolle des Europäischen Parlamentes sowie zum Kompetenzkatalog, so erscheinen die Vorgaben weniger überzeugend. Hier sah sich das Bundesverfassungsgericht eher in der Position, das Prinzip der Einzelermächtigung und das Subsidiaritätsprinzip weiter zu konkretisieren. Dieses Recht steht aber in erster Linie dem Rat oder jedenfalls dem EuGH zu. Noch kritischer sind die Entscheidungen zur Prüfungskompetenz nationaler Parlamente und vor allem zur ausschließlichen (Rest-)Kompetenz der Mitgliedstaaten zu bewerten. Hier fehlt es am Rechtsnotstand und an der durch die Entscheidung vermittelnden Rechtssicherheit. Gerade das Lissabon-Urteil ist eher geeignet, die sinnvolle Weiterentwicklung der Europäischen Union zum Stillstand zu zwingen, ${ }^{94}$ wenn die EU aus „Angst“" vor einem negativen Urteil des Bundesverfassungsgerichts vor weiteren Integrationsschritten zurückschreckt. ${ }^{95}$ Die tatsächlichen Folgen

91 In der Formulierung von $F$. Baur, Sozialer Ausgleich durch Richterspruch, JZ 1957, S. 195, 196: „,Der Richter wird an die Stelle des Gesetzgebers gerückt, ohne dass ihm dessen Hilfsmittel zur Verfügung stünden oder sein Urteil einem ähnlichen Gestaltungsprozeß unterläge wie das Gesetz.". S. E. Kramer (Fn. 82), S. 285; E. Pi-

92 S. etwa E. Kramer (Fn. 82), S. 284; A. Meyer-Hayoz, Strategische und taktische Aspekte der Fortbildung des
Rechts, JZ 1981, S. 417, 422.

93 EuGH, Rs. 4/73 (Nold), Sig. 1974, 491, Rn. 13.

94 D. Halberstam/C. Möllers, The German Con 1250 f.; C. Schönberger (Fn. 59), S. 1209.

95 C. Schönberger (Fn. 59), S. 1216. 
der Lissabon-Entscheidung auf europäischer Ebene wurden allerdings durch den Honeywell-Beschluss abgemildert. ${ }^{96}$

Auch erscheint es für die vorgestellten Fälle wenig überzeugend, die Untätigkeit des Gesetzgebers als Parameter hinzuzuziehen. Im nationalen Recht handelt der Gesetzgeber oft über Jahrzehnte nicht. ${ }^{97}$ Im Gegensatz dazu haben die Mitgliedstaaten als Herren der Verträge das Primärrecht in den letzten 20 Jahren sehr häufig geändert.

Das Bundesverfassungsgericht ist damit ein weiterer „Player“ der europäischen Rechtspolitik und spielt eine Rolle, die eigentlich in erster Linie dem europäischen Rat als Zusammenschluss von Vertretern der nationalen Regierungen gem. Art. 15 EUV zusteht.

Allerdings kann man auch für das Bundesverfassungsgericht streiten: So standen bei den jüngeren Entscheidungen zwar nicht die Grundrechte als Abwehrrechte im Raum. Jedoch war die Verfassung auch in jüngeren Entscheidungen berührt, sei es bei der Verletzung des Wahlrechts nach Art. $38 \mathrm{GG}^{98}$ oder des Demokratieprinzips nach Art. $20 \mathrm{GG}^{99}$. Zudem hatte in anderen Mitgliedstaaten das Staatsvolk die Möglichkeit, per Volksabstimmung über die Verträge abzustimmen. ${ }^{100}$ Weil diese Möglichkeit in Deutschland fehlt, ${ }^{101}$ müsse das Bundesverfassungsgericht die Möglichkeit haben, eine allgemeine Skepsis über eine Vertragserweiterung zum Ausdruck zu bringen. Das Bundesverfassungsgericht nutzte hier die Möglichkeit, die negative Stimmung in Worte zu fassen.

Schon vor Jahren wurde eine politische Diskussion der Völker über Europa gefordert; 102 das Bundesverfassungsgericht hat jüngst den Gedanken einer zwingenden Volksabstimmung ins Spiel gebracht. ${ }^{103}$ Über Eingriffe in den Identitätskern der

96 BVerfG, Beschl. v. 6.7.2010, BVerfGE 126, 286, Ls. 1, 304, Rn. 61 - Ultra-vires-Kontrolle Mangold: „wenn ein Kompetenzverstoß hinreichend qualifiziert ist"; C. Möllers, German Federal Constitutional Court, Constitutional ultra vires Review of European acts only under exceptional circumstances; Decision of 6 July 2010, 2 BvR 2661/06, Honeywell, European Constitutional Law Review 2011, S. 161.

97 S. etwa die Frage der culpa in contrahendo, V. Emmerich, in: Säcker/Rixecker (Fn. 81), §311, Rn. 37 und Fn. 87; die Absprachen im Strafprozess, s. dazu grundsätzlich positiv W. Beulke, Strafprozessrecht, 11. Aufl. 2010, Rn. 394; kritisch dagegen L. Meyer-Goßner, Strafprozessordnung, 55. Aufl. 2012, § 257c, Rn. 2 f.; $C$. Roxin/B. Schünemann, Strafverfahrensrecht, 27. Aufl. 2012, §44, Rn. $59 \mathrm{ff}$.

98 BVerfG, Urt. v. 30.6.2009, BVerfGE 123, 267, 330, Rn. 174 f. - Lissabon. Im Ergebnis ebenso bereits BVerfG, Urt. v. 12.10.1993, BVerfGE 89, 155, Ls. 1 - Maastricht. Dagegen ließe sich allerdings wiederum anführen, die Begründung über Art. 38 GG wäre nur ein „Kunstgriff“, ausführlich E. Brok/M. Selmayr (Fn. 33), S. 221 f.

99 Jüngst BVerfG, Urt. v. 12.9.2012, NJW 2012, 3145, 3150, Rn. 223 - ESM.

100 Anders als noch über den Europäischen Verfassungsvertrag stimmten über den Vertrag von Lissabon nur die Iren per Volksabstimmung ab, s. J. Leithäuser/N. Busse, Iren sagen ja zum Lissabonvertrag, Frankfurter Allgemeine Sonntagszeitung v. 4.10.2009, S. 1.

101 Die bayerische Staatsregierung plante einen Antrag auf bundesweite Volksentscheide zu grundlegenden europäischen Themen in den Bundesrat einzubringen, s. Pressemitteilung Nr. 396 der Bayerischen Staatsregierung v. 11.12.2012; Bayern will mehr Plebiszite, SZ v. 12.12.2012, S. 6. Der Erfolg eines solchen Antrags darf bezweifelt werden.

102 T. Möllers, Die Rolle des Rechts im Rahmen der europäischen Integration, 1999, S. 79 f.

BVerfG, Urt. v. 30.6.2009, BVerfGE 123, 26, 347 f., Rn. 228 ff. - Lissabon: „Das Grundgesetz ermächtigt die für Deutschland handelnden Organe nicht, durch einen Eintritt in einen Bundesstaat das SelbstbestimmungsVolkes in Gestalt der völkerrechtlichen Souveränität Deutschlands aufzugeben. Dieser

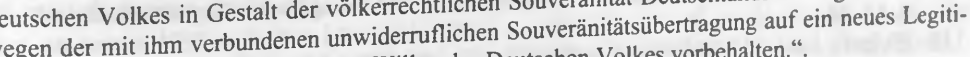
mationssubjekt allein dem unmittelbar erklärten Willen des Deutschen Volkes vorbehalten.“. 
Verfassung, insbesondere auch die „Ewigkeitsgarantie“ des Art. 79 Abs. 3 GG betreffend, könne nur das Volk als pouvoir constituant entscheiden. ${ }^{104}$ Dadurch sind die Grenzen parlamentarischen Handelns richtig definiert und auch die verfassungsgerichtliche Rechtsfortbildung wird eingeschränkt. Diese Abgrenzung gewinnt allerdings erst an Substanz, wenn die Identität der Verfassung bzw. der Inhalt des Art. 79 Abs. 3 GG konkretisiert werden - eine Aufgabe, die nach Huber wiederum dem Bundesverfassungsgericht obliegt. ${ }^{105}$ Damit allerdings maßt sich das Gericht ebenjene Kompetenz-Kompetenz an, die es dem EuGH entziehen möchte. Der Verfassungsgeber bezweckte mit Art. 79 Abs. 3 GG primär, die freiheitlich demokratische Grundordnung festzuschreiben und dem Zugriff durch den Gesetzgeber zu entziehen. ${ }^{106}$ Schon von Art. 1 und 20 GG will Art. 79 Abs. 3 GG nur die Grundsätze schützen. Nicht alles, was Menschenwürde oder Demokratieprinzip berührt, ist automatisch unzulässig. ${ }^{107}$ Der Verfassungsgeber war geprägt von den Erfahrungen der Weimarer Republik und des „Dritten Reiches“; er versuchte vor allem zu verhindern, dass auch die neue Verfassung ausgehöhlt würde und eine neue Diktatur entstünde. ${ }^{108}$ Die Entstehungsgeschichte zeigt, dass sich die Mütter und Väter des Grundgesetzes nur um die wichtigsten verfassungsrechtlichen Grundsätze sorgten; so fanden etwa die Wesensgehaltsgarantie (Art. 19 Abs. 2 GG) und die Garantien des Art. 19 Abs. 4 GG keinen Eingang in die Aufzählung des Art. 79 Abs. 3 GG. ${ }^{109}$ Erweiterungen der in Art. 79 Abs. 3 genannten Normen führen nicht zu einer Erweiterung der Ewigkeitsklausel selbst; 110 dies macht deutlich, dass der historische Wille des Verfassungsgebers über den Umfang der Klausel entscheidet. Das Bundesverfassungsgericht selbst betrachtet Art. 79 Abs. 3 GG als ,eng auszulegende Ausnahmevorschrift“; 111 diesen Ausnahmecharakter scheint es im europarechtlichen Kontext allerdings selbst nicht immer ernst zu nehmen.

\section{Formale Parameter zulässiger Rechtsfortbildung}

\section{a) Kooperationsverhältnis}

Die Solange-II-Entscheidung wurde als „Kooperationsangebot“ des Bundesverfassungsgerichts an den EuGH aufgefasst; ${ }^{112}$ das Gericht betonte dann in der Maastricht-Entscheidung selbst ein solches Verhältnis. ${ }^{113}$ Der Rechtsprechungsdialog

104 P. Huber, in: Möllers/Zeitler (Hrsg.), Europa als Rechtsgemeinschaft - Währungsunion und Schuldenkrise, 2013, S. 229, 238; H. Dreier, in: Dreier (Hrsg.), GG, 2. Aufl. 2006, Art. 79 III, Rn. 14.

105 P. Huber, in: Möllers/Zeitler (Fn. 104), S. 238.

106 H. Dreier, in: Dreier (Fn. 104), Art. 79 III, Rn. 5.

107 H. Dreier, in: Dreier (Fn. 104), Art. 79 III, Rn. 26.

108 M. Herdegen, in: Maunz/Dürig (Hrsg.), GG, 2012, Art. 79, Rn. 63 ,

109 M. Herdegen, in: Maunz/Dürig (Fn. 108), Art. 79, Rn. 67.

110 M. Herdegen, in: Maunz/Dürig (Fn. 108), Art. 79, Rn. 77; H. Dreier, in: Dreier (Fn. 104), Art. 79 III, Rn. 54.

111 BVerfG, Urt. v. 3.3.2004, BVerfGE 109, 279, 310 - großer Lauschangriff.

112 So etwa $P$. Kirchhof, Deutsches Verfassungsrecht und Europäisches Gemeinschaftsrecht, EuR-Beiheft 1991 ,

113 BVerfG, Urt. v. 12.10.1993, BVerfGE 89, 155, 156, 175 - Maastricht. 
zwischen nationalen und europäischen Gerichten führt immer wieder dazu, dass nationale Gerichte den EuGH überzeugen und dieser seine Rechtsprechung ändert. ${ }^{1 / 4}$ Einige nationale Verfassungsgerichte, darunter neben dem deutschen auch das polnische, üben im Rahmen dieses Dialogs einen größeren ,freundschaftlichen Druck" aus als andere und behalten sich gewisse Kompetenzen vor. ${ }^{115}$ So entschied der polnische Verfassungsgerichtshof im Jahr 2005:

., Die Mitgliedstaaten behalten das Recht zur Beurteilung, ob die GemeinschaftsRechtssetzungsorgane bei dem Erlass eines bestimmten Aktes im Rahmen der übertragenen Kompetenzen handelten und ob sie ihre Grundsätze gemäß den Grundsätzen der Subsidiarität und Verhältnismäßigkeit ausübten. Eine Überschreitung dieser Rahmen bewirkt, dass die außerhalb von ihnen erlassenen Akte nicht dem Grundsatz des Vorrangs des Gemeinschaftsrechts unterfallen. "I16

Ähnlich bemüht sich das Bundesverfassungsgericht, durch die ultra-vires-Lehre das letzte Wort in der Auslegung jedenfalls der Kompetenzen der Union zu behalten; ${ }^{17}$ dies widerspricht der Rechtsauffassung des EuGH, der sich selbst dazu befugt sieht. ${ }^{118}$ Die Drohung des Bundesverfassungsgerichts ist auch aufgrund der deutschen Position innerhalb der EU nicht zu unterschätzen. Es ist etwa im Zusammenhang mit den Zahlungsverpflichtungen rund um die Euro-Rettung kaum zu erwarten, dass der EuGH eine Entscheidung fällt, durch die Deutschland als Gläubiger ausfällt. Ferner kann der Verweis auf das BVerfG der deutschen Politik ermöglichen, bestimmte Entscheidungen zugunsten der verschuldeten Staaten abzulehnen. ${ }^{119}$ Damit nimmt das Bundesverfassungsgericht zwar keinen unmittelbaren Einfluss auf das europäische Recht, wohl aber auf die europäische Politik bzw. Willensbildung. In Anbetracht der aktuellen Entscheidungen erscheint der Begriff des Kooperationsverhältnisses daher allzu euphemistisch. Durch seine Entscheidungen gestaltet das Bundesverfassungsgericht aktiv das Europarecht mit - auch über die Grenzen der Bundesrepublik hinaus. ${ }^{120}$

114 T. von Danwitz, Kooperation der Gerichtsbarkeiten in Europa, ZRP 2010, S. 143, 145, verweist in diesem Zusammenhang auf EuGH, Rs. C-269/90 (TU München), Slg. 1991, I-5469 und EuGH, Rs. C-206/94 (Paletta II), Slg. 1996, I-2357 nach EuGH, Rs. C-45/90 (Paletta I), Slg. 1992, I-2423.

115 So G. Canivet, in: Cour de justice des Communautés européennes (Hrsg.), Actes du Colloque pour le cinquantième anniversaire des traités de Rome (1957-2007), 2007, Rn. 12.

116 Trybunal Konstytucyjny, Urt. v. 11.5.2005, AZ.K 18/04, in deutscher Übersetzung EuR 2006, S. $236,243$.

117 S. oben Fn. 65.

118 J. Bast/A. von Bogdandy, in: Grabitz/Hilf/Nettesheim (Fn. 14), Art. 5 EUV, Rn. 30; S. Pötters/J. Traut, Die ultra-vires-Kontrolle des BVerfG nach „Honeywell“ - Neues zum Kooperationsverhältnis von BVerfG und EuGH?, EuR 2011, S. 580, 583; EuGH, Rs. C-376/98 (Deutschland/Rat), Slg. 2000, I-8419.

119 M. Ruffert, Die europäische Schuldenkrise vor dem Bundesverfassungsgericht - Anmerkung zum Urteil vom 7. September 2011, EuR 2011, S. 842, 854.

120 C. Tomuschat sah bereits 1993 nach dem Maastricht-Urteil des BVerfG „Die Europäische Union unter der Aufsicht des Bundesverfassungsgerichts“, s. hierzu den gleichnamigen Aufsatz in der EuGRZ 1993, S. 489. 


\section{b) Loyalitätsprinzip und Vorlagepflicht}

Art. 4 Abs. 3 EUV normiert den Grundsatz der loyalen Zusammenarbeit zwischen EU und Mitgliedstaaten. ${ }^{121}$ Daraus folgt für die Mitgliedstaaten, dass sie nationales Recht, das gegen Europarecht verstößt, nicht anwenden. ${ }^{122}$

Das Verhältnis von EuGH und nationalen Gerichten wird dabei geprägt vom Auslegungsmonopol des EuGH für Europarecht sowie von der daraus folgenden Vorlagepflicht im Rahmen des Vorabentscheidungsverfahrens. In der CILFIT-Rechtsprechung ${ }^{123}$ führte der EuGH aus, die Vorlagepflicht bestünde bei Entscheidungserheblichkeit der Auslegungsfrage. Eine Vorlagepflicht besteht für letztinstanzliche Gerichte (Art. 267 Abs. 1, 3 AEUV), betrifft also auch das Bundesverfassungsgericht. Eine Vorlage des Bundesverfassungsgerichts an den EuGH gab es allerdings noch nie. ${ }^{124}$ Nach Ansicht des Bundesverfassungsrichters Gerhardt mangelt es in den Verfahren vor dem Bundesverfassungsgericht regelmäßig an der Entscheidungserheblichkeit, weil die deutsche und die europäische Rechtsordnung voneinander unabhängig seien. ${ }^{125}$

Das Unionsrecht ist nur durch individuelle Rechtsanwendungsbefehle (Zustimmungsgesetze) Teil der deutschen Rechtsordnung. ${ }^{126}$ Diese Zustimmungsgesetze unterliegen der Prüfung durch das Bundesverfassungsgericht. Dies bedeutet im Ergebnis, dass das Bundesverfassungsgericht über den Umweg der Zustimmungsgesetze die verfassungsrechtliche Grenze für den Vorrang des Unionsrechts in Deutschland prüft ${ }^{127}$ und damit das Auslegungsmonopol des EuGH durch Pflicht zur Kooperation (Art. 19 Abs. 1 UAbs. 2 EUV) einschränkt. ${ }^{128}$

$\mathrm{Ob}$ eine Unabhängigkeit der Rechtsordnungen tatsächlich existiert, sei dahingestellt; jedenfalls die Prüfung des (nationalen) Zustimmungsgesetzes ist unproblematisch. Dabei legt das Bundesverfassungsgericht aber mehr oder weniger offensichtlich auch Europarecht aus. Im Maastricht-Urteil etwa interpretiert das Bundesverfassungsgericht Art. F Abs. 3 EUV und kommt zu dem Schluss, es handle

121 Vgl. zu diesem Grundsatz EuGH, Rs. 230/81 (Luxemburg/Parlament), Slg. 1983, 225, Rn. 37.

122 EuGH, Rs. C-262/97 (Rijksdienst voor Pensioenen/Robert Engelbrecht), Slg. 2000, I-7321, Rn. 40; EuGH, Rs. C-144/04 (Mangold), Slg. 2005, I-9981, Rn. 77s. EuGH, Rs. C-144/04 (Mangold), Slg. 2005, I-9981, Rn. 77; EuGH, Rs. C-555/07 (Kücükdeveci), Slg. 2010, I-365, Rn. 51.

123 EuGH, Rs. 283/81 (CILFIT), Slg. 1982, 3415.

124 Sie könnte freilich im Rahmen der Entscheidung über Staatsanleihenankäufe durch die EZB erforderlich werden, s. M. Ruffert, Anmerkung zu EuGH, Rs. C-370/12, Urt. v. 27. November 2012 (Pringle), JZ 2013, S. 257, 259; ebenso D. Thym, Europarechtskonformität des Euro-Rettungsschirms (EuGH, Urt. v. 27.11.2012, Rs. C-370/12), JZ 2013, S. 259, 263, der kritisiert, dass das BVerfG mit der bisherigen Praxis der Nichtvorlage den Eindruck erwecke, ,, dass seine Deutung des Grundgesetzes der rechtliche Fixpunkt der Euro-Rettung sei“"

125 M. Gerhardt (Fn. 67), S. 162 f. Dagegen F. Mayer, Das Bundesverfassungsgericht und die Verpflichtung zur Vorlage an den Europäischen Gerichtshof zugleich Anmerkung zum Beschluss vom 22. November 2001-2 BvB 1-3/0I (NPD-Verbot), EuR 2002, S. 239. 243, der in der Auslegung des Europarechts durch das BVerfG

126 die Bestätigung der Entscheidungserheblichkeit sieht.

126 R. Streinz, in: Streinz (Fn. 36), Art. 288 AEUV, Rn. 39.

127 R. Streinz, in: Streinz (Fn. 36), Art. 288 AEUV, Rn. 39. 128 Streinz, in: Streinz (Fn. 36), Art. 288 AEUV, Rn. 39; BVerfG, Urt. v. 12.10.1993, BVerfGE 89, 155, 175 -
Maastricht. 
sich nicht um eine Befugnisnorm. ${ }^{129}$ In der Entscheidung über den Eilantrag gegen den ESM schreibt das Bundesverfassungsgericht der Bundesregierung vor, mittels eines Vorbehalts völkerrechtlich sicherzustellen, dass die deutsche Zahlungsverpflichtung durch den ESM nicht über 190 Mio. EUR ausgedehnt werden darf bzw. in diesem Fall keine deutsche Bindung besteht. ${ }^{130}$ Welche Auslegung der EuGH tatsächlich wählen würde, hätte er nach Inkrafttreten des Vertrages darüber zu entscheiden, ist unerheblich, solange Deutschland qua Vorbehalt eine Auslegung erzwingen kann. Im Eilverfahren besteht zudem keine Vorlagepflicht. Indem es einen Vorbehalt vorschreibt, entkommt das Bundesverfassungsgericht der Pflicht zur Vorlage an den EuGH: Bei Entscheidung des Hauptverfahrens wird dieser Vorbehalt bereits bestehen, sodass es für das Bundesverfassungsgericht nicht mehr darauf ankommt, wie der ESM tatsächlich (vom EuGH) ausgelegt wird. Zur Verdeutlichung: Wäre der ESM ohne Vorbehalt ratifiziert und das Bundesverfassungsgericht der Auffassung, dieser sei nur unter der Voraussetzung einer bestimmten Auslegung verfassungsgemäß, müsste das BVerfG dem EuGH vorlegen, damit dieser den ESM auslegen kann. Dazu wird es nun voraussichtlich nicht kommen.

\section{c) Nochmals: Judicial Activism und (unzulässiges) Spiel über die Bande}

Um ihre Meinung durchzusetzen, sind die nationalen Vertreter im Rat gezwungen, Mehrheiten zu suchen. Jetzt bleibt der trockene Beigeschmack, dass die Mehrheitsverhältnisse in den EU-Gremien verändert werden. Deutschland hatte bisher nur eine Stimme (was etwa jüngst mehrfach für die Stimmenverteilung in der EZB kritisiert wurde). Diese Stimmverteilung entsprach dem Grundgedanken der in der Union gleichberechtigten Völker, in der nicht die Größe des Mitgliedstaats oder gar seine Finanzmacht zählten.

Durch das Bundesverfassungsgericht erhält Deutschland nun aber nicht etwa nur eine zweite Stimme. Die Bindungswirkung der Entscheidungen des Bundesverfassungsgerichts gem. $\S 31 \mathrm{BVerfGG}$ führt zu einer unmittelbaren Beachtungspflicht durch Regierung und Parlament ${ }^{131}$ und damit faktisch zu einer Blockadehaltung, sollte man dem Willen des BVerfG nicht Rechnung tragen. Die Entscheidungen zeichnen sich dadurch aus, dass sie das europäische Recht mitgestalten, wie die obigen Entscheidungen verdeutlicht haben. Formal wird zwar immer wieder der Einschätzungsspielraum des Bundestages und damit der „Judicial Self-Restraint“ betont. ${ }^{132}$ Um die Umsetzung ihrer Vorgaben zu erreichen, droht das Gericht aber nicht nur mit der Unverbindlichkeit der Normen, sondern macht auch genaue Vorgaben über die Höhe der Zahlungsverpflichtung im Rahmen des ESM. ${ }^{133}$ Die Frage

H. Bethge, in: Maunz/Schmidt-Bleibtreu/Klein/Bethge (Fn. 62), § 31, Rn. 121.

Etwa auch jüngst BVerfG, Urt. v. 12.9.2012, 2 BvR 1390/12, Rn. 200 und $234=$ NJW 2012, 3145, 3147, 3151 - ESM.

133 BVerfG, Urt. v. 12.9.2012, NJW 2012, 3145, 3153, Rn. 253 - ESM. 
beispielsweise, ob die EZB Staatsanleihen kaufen darf oder nicht, ist in erster Linie eine politische, die im Rahmen der europäischen Willensbildung gestellt und beantwortet werden muss. Hier die EZB in ihren Handlungsspielräumen einzuschränken ist nicht nur politisch, sondern auch wirtschaftlich in hohem Maße unvernünftig, weil ihr dann Mechanismen genommen werden, um angemessen auf spekulierende Marktteilnehmer zu reagieren. Im Übrigen liegt die Auslegung der Vorschriften zur Währungsunion nun einmal in der Kompetenz des EuGH. Es wäre fatal, wenn das BVerfG im Hauptsacheverfahren zum ESM anders urteilen würde als der EuGH. ${ }^{134}$ Den deutschen Regierungsvertretern eröffnet sich außerdem die Möglichkeit des „Spiels über die Bande“: Wenn die Mehrheit politisch nicht erreichbar oder opportun ist, können sie den Ball gewissermaßen dem Bundesverfassungsgericht zuspielen, das die Aufforderung zur aktiven Mitgestaltung des Europarechts gern annimmt.

\section{Ausblick}

Die Entscheidung über den Eilantrag zum ESM steht am vorläufigen Ende der verfassungsgerichtlichen Entscheidungen zu Europa. Obgleich es sich um eine nationale Entscheidung handelte, der nur eine summarische Prüfung vorausging, fand das ESM-Urteil europa- und weltweit positive Beachtung. ${ }^{135}$ Die Pringle-Entscheidung des EuGH, ${ }^{136}$ dem eigentlich für die Auslegung von Unionsrecht zuständigen Gericht, genoss weit weniger Medienöffentlichkeit. ${ }^{137}$ Auch politisch hatte die Entscheidung des Bundesverfassungsgerichts europaweite Folgen: Den Vorbehalten, die es aussprach, schlossen sich die anderen Mitgliedstaaten an. ${ }^{138}$

Noch ist offen, ob auch die weiteren Urteile, die vom Bundesverfassungsgericht im Zusammenhang mit der Eurokrise zu erwarten sind, namentlich das HauptsacheUrteil zum ESM und die Entscheidung zur Europäischen Zentralbank, ebenso positiv aufgenommen werden. Außerdem bleibt abzuwarten, was das Bundesverfassungsgericht in Sachen EZB zu sagen hat. Nimmt man ernst, was das Bundesverfassungsgericht im ESM-Urteil entschied, sieht es für den Ankauf von Staatsanleihen durch die EZB schlecht aus. ${ }^{139}$

Ganz unstrittig wird man feststellen können, dass das Bundesverfassungsgericht die Grenzen unzulässiger Rechtsfortbildung im Spannungsfeld von deutschem und eu-

134 Der EuGH urteilte bislang über den ESM nur einmal, EuGH, Rs. C-370/12 (Thomas Pringle/Government of Ireland, Ireland, The Attorney General).

135 S. etwa H. Tory/G. Smith, German Court Clears Rescue Fund, www.wallstreetjournal.com, 12.9.2012.

136 EuGH, Rs. C-370/12 (Thomas Pringle/Government of Ireland, Ireland, The Attorney General).

137 Zurecht kritisch dazu M. Ruffert (Fn. 124), S. 257.

138 W. Mussler, Eurogruppe erfüllt Karlsruher Bedingung, FAZ v. 15.9.2012, S. 1.

139 BVerfG, Urt. v. 12.9.2012, NJW 2012, 3145, 3149, Rn. 220 - ESM: „Ein wesentliches Element zur unionsrechtlichen Absicherung der verfassungsrechtlichen Anforderungen aus Art. 20 Abs. 1 und Abs. 2 in Verbindung mit Art. 79 Ahs. 3 GG ist insoweit das Verbot monetärer Haushaltsfinanzierung durch die Europäische Sekundärmarkt unterschieden. S. Allerdings wurde hier nicht zwischen dem Ankauf von Staatsanleihen auf dem Primär- und 
ropäischem Verfassungsrecht weiter zieht als im Straf- und Zivilrecht. ${ }^{140}$ Der EuGH ist schon als Motor der Europäischen Gemeinschaft beschrieben worden, oft mit dem Unterton, er überschreite seine Kompetenzen. Dieser Beitrag sollte zeigen, dass auch das Bundesverfassungsgericht ein Motor der Europäischen Union ist und die Aufgabe, Lücken zu füllen oder unbestimmte Prinzipien zu konkretisieren, gern übernimmt: Grundrechte sind aus dem Unionsrecht inzwischen nicht mehr wegzudenken. Während die Kompetenzen der Union auch durch den Lissabon-Vertrag ausgeweitet wurden, sind die Wahrung des Prinzips der begrenzten Einzelermächtigung und eine starke Rolle der nationalen Parlamente zu immer wichtigeren Voraussetzungen einer funktionierenden und weitgehend akzeptierten Europäischen Union geworden. Die besprochenen Urteile lassen erwarten, dass das Bundesverfassungsgericht europarechtlich und -politisch wichtig bleibt, haben doch seine Vorgaben in der Vergangenheit häufig zu Gesetzesänderungen auf europäischer Ebene geführt. $\mathrm{Ob}$ hierbei der zur Begründung einer Lücke erforderliche Handlungsbedarf immer bestanden hat, mag man bezweifeln. Oder, um mit den Worten der Bundesverfassungsrichter Voßkuhle, Osterloh und Di Fabio zu schließen:

„Das Demokratieprinzip und das Funktionsgefüge des Grundgesetzes nähmen nachhaltig Schaden, könnte sich die Rechtsprechung immer dann über die eindeutige gesetzgeberische Entscheidung hinwegsetzen, wenn sie die Konsequenzen einer solchen Entscheidung als ,unzweckmäßig“ ansieht und der Gesetzgeber nach entsprechender richterlicher Anmahnung nicht wie gewünscht handelt. Klar erkennbare gesetzgeberische Regelungskonzepte sind vom Richter zu respektieren". ${ }^{141}$

140 S. die Nachweise bei T. Möllers, Ein Vier-Stufen-System zur Rationalisierung der Grenze zulässiger Rechtsfortbildung, in: Stelmach/Schmidt (Fn. 77), S. $121 \mathrm{ff}$.

141 Sondervotum der Richter Voßkuhle/Osterloh/Di Fabio, in: BVerfG, Beschl. v. 15.1.2009, BVerfGE 122, 248, 285, Rn. 103 - Rügeverkümmerung. 